\title{
Plasma Enhanced Atomic Layer Deposition on Powders
}

\section{Geert Rampelberg, Christophe Detavernier \\ Department of Solid State Sciences, Ghent University, Krijgslaan 281 (S1), B-9000 Ghent, Belgium \\ Geert.Rampelberg@UGent.be}

Surface engineering of micro- and nanoparticles is of great importance in fields such as catalysis, energy and sensing. For many of these applications particles are required with different bulk and surface properties. A popular technique to achieve this is to coat the particle surface with a nanometer thick layer. Only a few techniques have been explored for depositing such thin conformal coatings. Chemical vapor deposition (CVD) has been used extensively for this purpose, but suffers from a lot of disadvantages, such as imperfect control over layer thickness and uniformity of the coating over all individual particles, particle agglomeration and formation of additional undesired particles due to gas phase reactions between the CVD reactants. In contrast, atomic layer deposition (ALD) is known as a reliable technique for covering complex 3D objects with ultrathin conformal coatings. However, to perform ALD on large quantities of powders, the individual particles need to be fluidized or agitated. Fluidized bed reactors are most often used for ALD on particles, but this reactor concept does not seem to be compatible with plasma enhanced ALD, which is advantages for e.g. coating on temperature sensitive polymer particles or deposition of metals and metal nitrides.

In this work, a rotary reactor was used to agitate particles, enabling the deposition of conformal coatings by thermal and plasma-enhanced ALD. Particles ranging from nanometer size to millimeter size were successfully coated with layers of $\mathrm{Al}_{2} \mathrm{O}_{3}, \mathrm{TiO}_{2}$, AIN and TiN. ${ }^{1}$ In-situ mass spectroscopy confirmed that ALD was performed by detecting the expected reaction products. By monitoring the formation of these reaction products over time, it was possible to optimize precursor and reactant usage, which is linearly dependent on the effective surface area of the particles. In the case of plasma enhanced ALD, in-situ optical emission spectroscopy confirmed the mass spectroscopy data. X-ray fluorescence revealed the expected linear relationship between the amount of ALD cycles and the deposited amount of material, while X-ray photo-electron spectroscopy was used to confirm the composition and purity of the coatings. Transmission electron spectroscopy finally showed that the individual particles were coated uniformly and conformally. Our results prove that the proposed rotary reactor enables conformal deposition on nano- and micropowders by thermal and plasma enhanced ALD. In this way, surface engineering of such particles can be achieved.

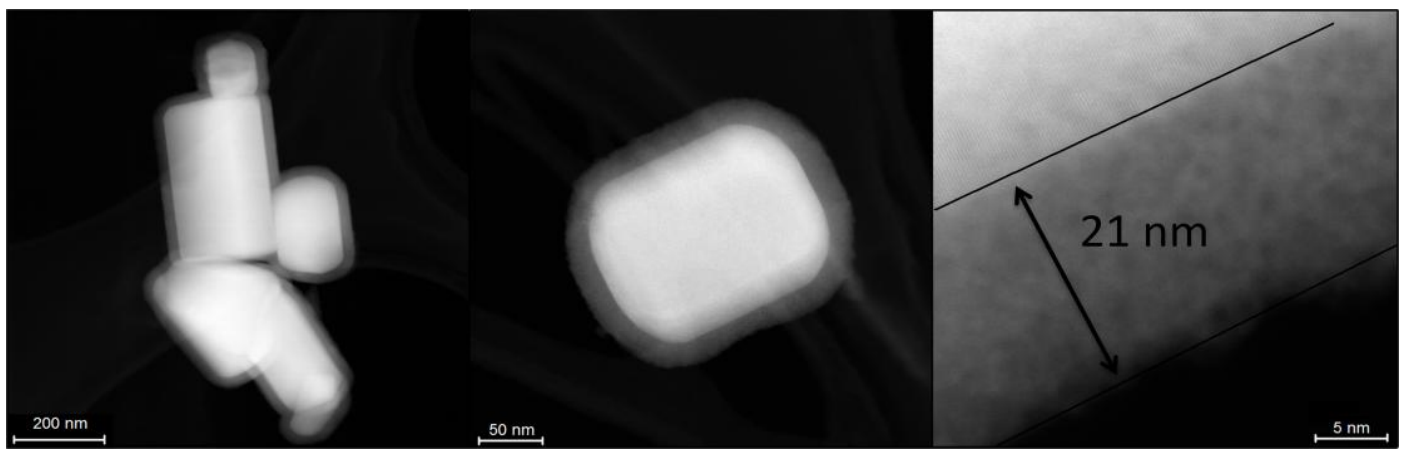

Figure 1: TEM picture of $\mathrm{ZnO}$ nanopowder coated with 300 plasma enhanced ALD cycles of TiN.

[1] D. Longrie et al., Surface \& Coating Technology 213 (2013) 183-191 